\title{
O TEOR DE CÁLCIO NA CASCA É INDICATIVO DA SUSCETIBILIDADE AO "BITTER PIT" EM MAÇÃ̃ 'FUJI'
}

\author{
CASSANDRO VIDAL TALAMINI DO AMARANTE ${ }^{2}$, PAULO ROBERTO ERNANI ${ }^{3}$, \\ CRISTIANO ANDRÉ STEFFENS ${ }^{4}$, LUIZ CARLOS ARGENTA ${ }^{5}$
}

RESUMO - Este trabalho objetivou identificar os atributos minerais na casca e na polpa, relacionados à severidade de "bitter pit" em maçãs 'Fuji'. Os frutos foram colhidos em pomar comercial do município de Lages-SC, e armazenados durante quatro meses em câmara fria convencional $\left(0 \pm 0,4^{\circ} \mathrm{C}\right.$ e $90-95 \%$ de umidade relativa). Posteriormente, foram separadas em quatro lotes, de acordo com a severidade de "bitter pit": nula (0 lesão/fruto), baixa (1-2 lesões/fruto), moderada (3-4 lesões/fruto) e alta (5-16 lesões/fruto), com 15 frutos (repetições) em cada nível de severidade. Os frutos de cada lote foram analisados individualmente quanto aos teores de $\mathrm{Ca}, \mathrm{Mg}, \mathrm{K}$ e N , na casca e na polpa. $\mathrm{Na}$ análise univariada dos teores minerais da polpa, observou-se aumento nos níveis de K e da relação K/Ca com o aumento na severidade de "bitter pit", apesar da ausência de diferenças quanto aos teores de $\mathrm{Ca}$. $\mathrm{Na}$ casca, os teores de $\mathrm{Ca}$ e de $\mathrm{N}$ diminuíram, e as relações $\mathrm{K} / \mathrm{Ca}, \mathrm{Mg} / \mathrm{Ca}$ e $(\mathrm{K}+\mathrm{Mg}) / \mathrm{Ca}$ aumentaram com o incremento da severidade do distúrbio. Pela análise canônica discriminante (análise multivariada), observou-se que a melhor separação entre níveis de severidade de "bitter pit" foi obtida com os teores de Ca na casca. Assim, para maçãs 'Fuji', é mais seguro fazer previsão da ocorrência de "bitter pit" durante a armazenagem, com base nos teores de Ca na casca do que na polpa. Frutos com teor de Ca na casca $\leq 240 \mathrm{mg} \mathrm{kg}^{-1}$ de matéria fresca são suscetíveis a esse distúrbio.

Termos para indexação: Malus domestica, qualidade de fruto, nutrição mineral, distúrbio fisiológico, análise multivariada.

\section{SKIN CALCIUM CONTENT IS INDICATIVE OF BITTER PIT SUSCEPTIBILITY IN 'FUJI’ APPLES}

\begin{abstract}
This study was carried out to identify the mineral attributes in the flesh and skin tissues better related to bitter pit severity in 'Fuji' apples. Fruits were harvested in a commercial orchard in Lages, Santa Catarina (SC), Brazil, and stored for four months in a conventional cold storage (at $0 \pm 0.4^{\circ} \mathrm{C}$ and 90 $95 \% \mathrm{RH})$. Subsequently, fruits were divided into four lots according to the level of bitter pit severity: none (0 pit/fruit), low (1-2 pits/fruit), moderate (3-4 pits /fruit), and high (5-16 pits /fruit). There were 15 fruit replicates for each level of bitter pit severity. Mineral analysis of $\mathrm{Ca}, \mathrm{Mg}, \mathrm{K}$, and $\mathrm{N}$ in the skin and in the flesh tissues were performed on individual fruits of each severity level. The univariate analysis in the fresh tissue showed an increase of $\mathrm{K}$ content and of the $\mathrm{K} / \mathrm{Ca}$ ratio with the increase of bitter pit severity, despite of no difference of $\mathrm{Ca}$ contents. In the skin, $\mathrm{Ca}$ and $\mathrm{N}$ contents reduced, and $\mathrm{K} / \mathrm{Ca}, \mathrm{Mg} / \mathrm{Ca}$, and $(\mathrm{K}+\mathrm{Mg}) /$ $\mathrm{Ca}$ ratios increased with the increase of bitter pit severity. However, the canonical discriminant analysis (multivariate analysis) showed that the best segregation between levels of bitter pit severity was achieved with the Ca content in the skin. Therefore, for 'Fuji' apples, it is safer to predict the occurrence of bitter pit during cold storage by assessing the Ca content in the skin rather than the in flesh. Fruit with Ca content in the skin $\leq 240 \mathrm{mg} \mathrm{kg}^{-1}$ of fresh matter are susceptible to bitter pit.
\end{abstract}

Index terms: Malus domestica; fruit quality, mineral nutrition, physiological disorder, multivariate analysis.

\footnotetext{
'(Trabalho 009-10). Recebido em: 04-01-2010. Aceito para publicação em: 05-08-2010.

${ }^{2} \mathrm{Ph}$.D., bolsista de produtividade em pesquisa do CNPq. Professor do Departamento de Agronomia, Centro de Ciências Agroveterinárias (CAV), Universidade do Estado de Santa Catarina (UDESC), CEP 88520-000, Lages-SC. Autor para correspondência. E-mail: amarante@cav.udesc.br

${ }^{3}$ Ph.D., bolsista de produtividade em pesquisa do CNPq. Professor do Departamento de Solos e Recursos Naturais, CAV/UDESC, Lages-SC. E-mail: a2pre@cav.udesc.br

${ }^{4}$ Dr., Professor do Departamento de Agronomia, CAV/UDESC, Lages-SC. E-mail: steffens@cav.udesc.br

${ }^{5}$ Dr., Pesquisador da Empresa de Pesquisa Agropecuária e Extensão Rural de Santa Catarina (Epagri), Estação Experimental de Caçador. Rua Abílio Franco, 1500, Bairro Bom Sucesso, CEP 89500-000, Caçador-SC. E-mail: argenta@epagri.sc.gov.br
} 


\section{INTRODUÇÃO}

O "bitter pit" é um dos principais distúrbios fisiológicos que depreciam a qualidade de maçãs em todos os países produtores (FERGUSON; WATKINS, 1989; SAURE, 2005). Apesar de este distúrbio desenvolver-se durante a fase de frigoconservação, suas causas e fatores predisponentes estão presentes no pomar, podendo, em casos extremos, os sintomas aparecerem antes da colheita (FAUST; SHEAR, 1968; FERGUSON; WATKINS, 1989). O sintoma primário é uma discreta mancha na polpa da maçã, de coloração escura, que se torna desidratada com o tempo, criando assim pequenas depressões na epiderme do fruto (FERGUSON; WATKINS, 1989).

No Brasil, a relação entre composição mineral e ocorrência de "bitter pit" foi estudada em maçãs 'Gala', 'Fuji', 'Catarina' e 'Golden Delicious' (ARGENTA; SUZUKI, 1994; NACHTIGALL; FREIRE, 1998; AMARANTE et al., 2006a e 2006b). O baixo conteúdo de Ca nos frutos é o principal fator predisponente à ocorrência de "bitter pit" (FERGUSON; WATKINS, 1989; AMARANTE et al., 2005; SAURE, 2005). A deficiência de $\mathrm{Ca}$ pode reduzir a integridade da parede celular e comprometer a permeabilidade seletiva das membranas celulares, resultando em injúria e necrose dos tecidos (FERGUSON; WATKINS, 1989; POOVAIAH, 1986; POOVAIAH et al., 1988). Além disto, o Ca tem importante papel regulatório do metabolismo celular de frutos (SAURE, 2005). A incidência de "bitter pit" também pode ser consequência de elevados valores das relações K/ $\mathrm{Ca},(\mathrm{K}+\mathrm{Mg}) / \mathrm{Ca}$ e N/Ca nos frutos (FAUST; SHEAR, 1968; FERGUSON; WATKINS, 1989; ARGENTA; SUZUKI, 1994; NACHTIGALL; FREIRE, 1998; AMARANTE et al., 2006a e 2006b).

O método normalmente empregado de amostragem de tecidos de maçãs para a análise dos conteúdos de $\mathrm{Ca}, \mathrm{Mg}, \mathrm{K}$ e $\mathrm{N}$ consiste na retirada de uma fatia longitudinal (menos pedúnculo e semente), contendo tecidos de casca e polpa. Todavia, o teor de $\mathrm{Ca}$ varia muito em diferentes partes do fruto. $\mathrm{O}$ teor de $\mathrm{Ca}$, em uma seção radial, é superior na casca e no centro do fruto, e inferior no córtex, sendo que os menores teores estão na parte externa do córtex, onde justamente ocorre o "bitter pit" (TERBLANCHE et al., 1979). Existe ainda um gradiente longitudinal, onde o teor de $\mathrm{Ca}$ diminui da região do pedúnculo para a região do cálice, razão pela qual o sintoma normalmente se manifesta na porção calicinar dos frutos, logo abaixo da casca (LEWIS; MARTIN, 1973; FERGUSON; WATKINS, 1983). Portanto, o método tradicional de amostragem (fatia longitudinal de casca+polpa), envolvendo tecidos com níveis minerais bastante diferenciados, pode reduzir a capacidade de predição do risco de "bitter pit". Desta forma, a análise do conteúdo de Ca na casca do fruto (e suas relações com $\mathrm{K}, \mathrm{Mg}$ e N) apresenta maior valor na predição de ocorrência de "bitter pit" em relação à análise do tecido da polpa (AMARANTE et al., 2005, 2006a, 2006b).

Este trabalho objetivou identificar os atributos minerais na casca e na polpa relacionados à severidade de "bitter pit" em maçãs 'Fuji'.

\section{MATERIAL E MÉTODOS}

Maçãs 'Fuji', oriundas de um pomar comercial localizado no município de Lages-SC, com histórico de elevada incidência de "bitter pit", foram armazenadas durante quatro meses em câmara fria convencional (temperatura de $0 \pm 0,4^{\circ} \mathrm{C}$ e $90-95 \%$ de umidade relativa), na safra de 2007/2008. Após este período, os frutos foram retirados do armazenamento refrigerado e divididos em quatro lotes, com diferentes níveis de severidades ao "bitter pit": 1$)$ nula (0 lesão/fruto); 2) baixa (1-2 lesões/fruto); 3 ) moderada (3-4 lesões/fruto); e 4) alta (5-16 lesões/fruto). Frutos individuais de cada lote (representando os diferentes níveis de severidades ao "bitter pit") foram analisados quanto aos teores de $\mathrm{Ca}, \mathrm{K}, \mathrm{Mg}$ e $\mathrm{N}$ nos tecidos da casca e da polpa. O delineamento experimental foi o inteiramente casualizado, com 15 repetições, sendo que cada repetição correspondeu a um fruto.

Os frutos foram lavados com água destilada antes da remoção dos tecidos destinados à análise mineral. Foi efetuada a remoção da casca de toda a superfície do fruto e utilizado todo o tecido da polpa (região do córtex, exceto os tecidos de origem carpelar, da região central) de cada fruto. Os tecidos da casca e da polpa dos frutos foram digeridos a $350^{\circ} \mathrm{C}$, usando-se uma mistura de ácido sulfúrico concentrado $\left(\mathrm{H}_{2} \mathrm{SO}_{4}\right)$ e água oxigenada $\left(\mathrm{H}_{2} \mathrm{O}_{2}, 30\right.$ volumes $)$. $\mathrm{O}$ $\mathrm{N}$ foi determinado pelo método semimicro-Kjeldahl, como descrito por Tedesco et al. (1995), e os demais nutrientes $(\mathrm{K}, \mathrm{Ca}$ e $\mathrm{Mg}$ ) foram determinados por espectrofometria de emissão induzida por plasma. Os teores dos diferentes elementos foram expressos em $\mathrm{mg} \mathrm{kg}^{-1}$ de matéria fresca.

Os dados obtidos foram submetidos à análise de variância e teste de Tukey $(P \leq 0,05)$, utilizando o programa SAS (SAS Institute, 2002).Os dados foram também submetidos à análise canônica discriminante (ACD), visando a identificar o tecido do fruto (casca ou polpa), bem como os atributos 
nutricionais que melhor permitem discriminar diferenças quanto ao grau de severidade ao "bitter pit". O coeficiente da taxa de discriminação paralela (TDP) foi usado para avaliar o efeito de separação, gerada pelos atributos nutricionais, entre os níveis de severidade estudados. O TDP é obtido através do produto entre valores dos coeficientes canônicos padronizados (CCP) e os valores dos coeficientes de correlação canônica (r). Para o coeficiente da TDP, valores positivos para atributos minerais indicam efeito de separação entre os níveis de severidade de "bitter pit". Quanto maiores os valores de TDP para os atributos minerais, maior o peso de separação entre níveis de severidade de 'bitter pit'. Valores negativos de TDP expressam efeito de supressão do atributo mineral na separação entre os níveis de severidade do distúrbio, ou seja, expressam semelhanças entre os níveis. Os valores médios de CCP de frutos correspondentes aos diferentes níveis de severidade ao "bitter pit" foram comparados através do teste de Tukey $(P \leq 0,05)$, conforme descrito por Amarante et al. (2006a).

\section{RESULTADOS E DISCUSSÃO}

\section{Análise univariada dos atributos minerais}

Para os teores de $\mathrm{Ca}$ e $\mathrm{N}$ e a relação N/Ca da polpa, não houve diferenças significativas entre os frutos com diferentes níveis de severidade de "bitter pit". Entretanto, frutos com nível de severidade moderada e alta de "bitter pit" apresentaram maiores teores de K na polpa, em relação aos frutos sem "bitter pit", o que também resultou em maiores valores das relações $\mathrm{K} / \mathrm{Ca},(\mathrm{K}+\mathrm{Mg}) / \mathrm{Ca}$ e $(\mathrm{K}+\mathrm{Mg}+\mathrm{N}) / \mathrm{Ca}$. Os níveis de $\mathrm{Mg}$ e a relação $\mathrm{Mg} / \mathrm{Ca}$ da polpa não foram crescentes em relação aos níveis de "bitter pit". A maior média de teor de $\mathrm{Mg}$ foi encontrada em frutos com baixa severidade deste distúrbio, seguido por frutos com alta severidade (Tabela 1).

$\mathrm{Na}$ casca, os teores de Ca foram significativamente maiores nos frutos sem "bitter pit" em relação àqueles com ocorrência do distúrbio. Os teores de $\mathrm{N}$ na casca foram maiores nos frutos com severidade nula e mais baixa do que naqueles com níveis moderado e alto. Em razão do aumento nos teores de $\mathrm{Ca}$ e de $\mathrm{N}$ na casca com a redução na severidade de "bitter pit", a relação N/Ca na casca não diferiu entre frutos com níveis de severidade nula e alta. Não houve diferença quanto aos teores de $\mathrm{K}$ na casca entre frutos com diferentes níveis de severidade ao "bitter pit”. Assim como na polpa, os teores de Mg na casca não foram diretamente relacionados ao aumento nos níveis de severidade ao "bitter pit". Os valores das relações $\mathrm{Mg} / \mathrm{Ca}, \mathrm{K} / \mathrm{Ca},(\mathrm{K}+\mathrm{Mg}) / \mathrm{Ca}$ e $(\mathrm{K}+\mathrm{Mg}+\mathrm{N}) / \mathrm{Ca}$ na casca apresentaram um acréscimo com o aumento na severidade ao "bitter pit", porém refletindo a diminuição nos teores de Ca na casca com o aumento na severidade do distúrbio.

Terblanche (1981) observa a necessidade de teores de $\mathrm{K}$ na polpa fresca serem menores do que $950 \mathrm{mg} \mathrm{kg}^{-1}$ a fim de minimizar a incidência de "bitter pit" em maçãs. Os resultados obtidos no presente estudo confirmam essa observação, pois frutos sem "bitter pit" apresentaram teor médio de K na polpa inferior a $950 \mathrm{mg} \mathrm{kg}^{-1}$; frutos com níveis de incidência de distúrbio de baixo a alto tiveram concentração de $\mathrm{K}$ na polpa acima desse valor (Tabela 1 ). Todavia, em maçãs 'Gala' e 'Catarina', não foi observado aumento nos teores de $\mathrm{K}$ e na relação $\mathrm{K} / \mathrm{Ca}$, tanto na polpa como na casca, com o aumento no nível de severidade de "bitter pit" (AMARANTE et al., 2006a e 2006b). Contudo, maçãs 'Fuji' com relação $(\mathrm{K}+\mathrm{Mg}) / \mathrm{Ca}$ no tecido da polpa $\geq 40$ apresentaram "bitter pit" (Tabela 1), valor este pouco maior do que $\geq 32,5$, encontrado em trabalhos anteriores para maçãs 'Golden Delicious' (NACHTIGALL; FREIRE, 1998) e 'Gala' (AMARANTE et al., 2006a). Argenta e Suzuki (1994) verificaram menor incidência de "bitter pit" em maçãs 'Gala' com relação $(\mathrm{K}+\mathrm{Mg}) / \mathrm{Ca}$ menor do que 27 no tecido de casca+polpa. Considerando que os autores amostraram casca+polpa, e que a casca apresenta menor valor de $(\mathrm{K}+\mathrm{Mg}) / \mathrm{Ca}$ em relação à polpa (Tabela 1), é esperado um valor menor desta relação quando realizada a amostragem de casca+polpa em comparação à amostragem apenas de polpa.

Amarante et al. (2006a) observaram "bitter pit” em maçãs 'Gala' apresentando, na casca, valores das relações $\mathrm{K} / \mathrm{Ca} \geq 12,4, \mathrm{Mg} / \mathrm{Ca} \geq 2,0,(\mathrm{~K}+\mathrm{Mg}) /$ $\mathrm{Ca} \geq 14,3$ e $(\mathrm{K}+\mathrm{Mg}+\mathrm{N}) / \mathrm{Ca} \geq 18,4$. Estes valores foram superiores aos observados em maçãs 'Fuji' no presente trabalho, nas quais frutos com "bitter pit" apresentaram, na casca, valores das relações $\mathrm{K} / \mathrm{Ca} \geq$ 9,2, $\mathrm{Mg} / \mathrm{Ca} \geq 1,6(\mathrm{~K}+\mathrm{Mg}) / \mathrm{Ca} \geq 10,8$ e $(\mathrm{K}+\mathrm{Mg}+\mathrm{N}) /$ $\mathrm{Ca} \geq 15,3$ (Tabela 1). A relação $\mathrm{N} / \mathrm{Ca}$ na casca de maçãs 'Fuji' com "bitter pit" foi superior a 4,0 (Tabela 1), similar aos resultados obtidos em maçãs 'Gala' (AMARANTE et al., 2006a). Em maçãs 'Golden Delicious', frutos com relação $(\mathrm{K}+\mathrm{Mg}) / \mathrm{Ca}$ na casca superior a 7,9, em base seca, apresentam maior ocorrência de "bitter pit" (NACHTIGALL; FREIRE, 1998).

Os resultados obtidos mostram que os valores críticos de teores de Ca e das suas relações com K, Mg e N em maçãs 'Fuji' dependem da parte do fruto amostrada. A análise univariada dos dados mostrou redução nos teores de $\mathrm{Ca}$ e $\mathrm{N}$ na casca, e aumento nos teores de $\mathrm{K}$ na polpa, com o aumento na severidade de "bitter pit". Em razão disso, as relações 
de $\mathrm{K} / \mathrm{Ca}$ e $\mathrm{Mg} / \mathrm{Ca}$ na casca, e a relação de $\mathrm{K} / \mathrm{Ca}$ na polpa, foram maiores em frutos que apresentaram maior severidade de "bitter pit" (Tabela 1).

\section{Análise multivariada dos atributos minerais}

$\mathrm{Na}$ análise multivariada, não foram considerados os teores isolados dos elementos $\mathrm{Mg}, \mathrm{K}$ e N na casca e na polpa, já que estes não apresentam clara relação com a ocorrência de "bitter pit" em maçãs (FAUST; SHEAR, 1968; FERGUSON; WATKINS, 1989; AMARANTE et al., 2006a e 2006b). Em razão disso, apenas os teores de $\mathrm{Ca}$, e suas relações com $\mathrm{Mg}, \mathrm{K}$ e N, nos tecidos da casca e da polpa, foram considerados nesta análise.

$\mathrm{Na}$ análise canônica discriminante (ACD), a primeira função discriminante canônica explicou $89 \%$ da variação total, enquanto a segunda e a terceira funções explicaram apenas $7,4 \%$ e $3,6 \%$, respectivamente. Como a primeira função discriminante canônica (função canônica discriminante $1 ; \mathrm{FCD}_{1}$ ) explicou a maior parte da variação total, mostrando que a separação entre frutos com diferentes níveis de severidade ao "bitter pit" é basicamente unidirecional, apenas esta foi considerada na análise multivariada dos dados obtidos.

O teste estatístico multivariado Wilks Lambda mostrou diferença altamente significativa $(P<0,0001)$ entre níveis de severidade ao "bitter pit" para a $\mathrm{FCD}_{1} \cdot \mathrm{AFCD}_{1}$ apresentou uma correlação canônica de 0,9268 , indicando elevada associação entre os atributos minerais estudados e os níveis de severidade ao "bitter pit".

$\mathrm{O}$ teor de $\mathrm{Ca}$ na casca apresentou o maior valor de taxa de discriminação paralela - TDP (Tabela 2), indicando que, dentre todos os atributos nutricionais avaliados, nos tecidos da casca e da polpa, este é o que melhor discrimina maçãs 'Fuji' com diferentes níveis de severidade ao "bitter pit". As relações $\mathrm{Mg} / \mathrm{Ca}$ e $\mathrm{K} / \mathrm{Ca}$ na casca também apresentam altos valores de TDP, embora menores do que aquele para o teor de Ca na casca, indicando que essas relações possuem menor grau de importância na discriminação de frutos quanto ao risco de "bitter pit". Na polpa, o teor isolado de $\mathrm{Ca}$, bem como suas relações com os demais elementos minerais, não se mostrou relevante na discriminação entre lotes de frutos com e sem "bitter pit". Isto confirma observações realizadas por outros autores, mostrando que a amostragem do tecido da casca é mais indicada para a análise mineral, visando a discriminar maçãs quanto à suscetibilidade ao "bitter pit” (AMARANTE et al., 2005, 2006a, 2006b).

Diversos autores, trabalhando com amostragem de casca+polpa em maçãs, mostraram que relações entre $\mathrm{Ca}$ e os elementos $\mathrm{Mg}$ e $\mathrm{K}$ são melhores indicadores de suscetibilidade ao "bitter pit" do que os teores isolados de Ca (FAUST; SHEAR, 1968; FERGUSON; WATKINS, 1989; ARGENTA; SUZUKI, 1994; NACHTIGALL; FREIRE, 1998). Mesmo em trabalhos envolvendo amostragem individual dos tecidos da casca e da polpa, também foi observado melhor segregação entre frutos com diferentes níveis de severidade de "bitter pit", quando consideradas as relações de $\mathrm{Ca}$ com K e Mg. Em maçãs 'Gala' e 'Catarina', frutos com valores altos das relações $\mathrm{K} / \mathrm{Ca}$ e $\mathrm{Mg} / \mathrm{Ca}$ no tecido da casca, respectivamente, apresentaram maior severidade de "bitter pit" (AMARANTE et al., 2006a e 2006b). Em maçãs 'Fuji', apenas o teor de Ca na casca mostrou-se o melhor indicador da suscetibilidade dos frutos ao "bitter pit". Portanto, para cada cultivar de maçã, existe um atributo mineral mais indicado para avaliar o risco de ocorrência de "bitter pit".

A representação gráfica entre os coeficientes canônicos padronizados (CCP) das funções canônicas discriminantes 1 e 2 mostra nítida separação entre frutos com diferentes níveis de severidade de "bitter pit" ao longo da $\mathrm{FCD}_{1}$ (Figura 1). Além disso, houve diferença altamente significativa $(P<0,0001)$ entre os valores médios de CCP da $\mathrm{FCD}_{1}$ entre os quatro grupos de severidade ao "bitter pit" (Tabela 3). Frutos com menores severidades de "bitter pit" apresentaram maiores valores médios de CCP para a $\mathrm{FCD}_{1}$, principalmente como resultado dos maiores teores de Ca na casca.

Os resultados obtidos demonstram que a quantificação da severidade de "bitter pit" em maçãs 'Fuji' não deve ser baseada na análise mineral de polpa+casca, pois esses tecidos apresentam níveis minerais bastante diferenciados. Segundo Ferguson e Watkins (1989) e Saure (2005), a manifestação das manchas de "bitter pit" ocorre em grupos específicos de células, que apresentam baixos níveis de $\mathrm{Ca}$, relativamente aos altos níveis de $\mathrm{Mg}, \mathrm{K}$ e N. Dessa forma, como no presente trabalho foi efetuada a remoção de toda a casca e de todo o tecido da polpa dos frutos, foram amostrados em grande parte tecidos com conteúdo mineral não predisponente à ocorrência de "bitter pit", mesmo na casca. No entanto, a análise multivariada demonstrou que é possível obter boa discriminação entre maçãs 'Fuji' com diferentes níveis de severidade ao "bitter pit", com base nos teores de Ca da casca, mas não da polpa dos frutos. Observou-se que frutos com teores de Ca no tecido da casca iguais ou inferiores a $240 \mathrm{mg}$ $\mathrm{kg}^{-1}$ de matéria fresca são suscetíveis ao "bitter pit". 
TABELA 1 - Atributos minerais na polpa e na casca de maçãs 'Fuji' com diferentes níveis de severidade de "bitter pit".

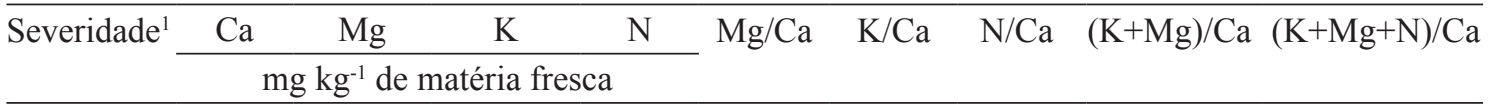

\begin{tabular}{lccccccccc}
\hline & \multicolumn{8}{c}{ Polpa } \\
Nula & $29,7^{\mathrm{ns}}$ & $56,6 \mathrm{c}^{2}$ & $922,7 \mathrm{c}$ & $224,9^{\mathrm{ns}}$ & $2,0 \mathrm{~b}$ & $32,8 \mathrm{~b}$ & $7,8^{\mathrm{ns}}$ & $34,8 \mathrm{~b}$ & $42,7 \mathrm{~b}$ \\
Baixa & 28,5 & $75,8 \mathrm{a}$ & $982,2 \mathrm{bc}$ & 204,2 & $2,8 \mathrm{a}$ & $37,1 \mathrm{ab}$ & 7,8 & $39,9 \mathrm{ab}$ & $47,7 \mathrm{ab}$ \\
Moderada & 25,7 & $53,8 \mathrm{c}$ & $1078,2 \mathrm{ab}$ & 218,0 & $2,3 \mathrm{ab}$ & $46,1 \mathrm{a}$ & 9,4 & $48,4 \mathrm{a}$ & $57,8 \mathrm{a}$ \\
Alta & 27,2 & $66,2 \mathrm{~b}$ & $1173,3 \mathrm{a}$ & 237,2 & $2,6 \mathrm{ab}$ & $46,8 \mathrm{a}$ & 9,3 & $49,5 \mathrm{a}$ & $58,8 \mathrm{a}$ \\
\hline CV (\%) & 27,2 & 20,1 & 19,6 & 23,7 & 37,9 & 38,1 & 37,8 & 37,1 & 35,8 \\
\hline \multicolumn{1}{c}{} & & \multicolumn{7}{c}{ Casca } \\
Nula & $291,2 \mathrm{a}$ & $323,1 \mathrm{~b}$ & $2155,1^{\mathrm{ns}}$ & $1140,7 \mathrm{a}$ & $1,1 \mathrm{c}$ & $7,5 \mathrm{~d}$ & $4,0 \mathrm{~b}$ & $8,7 \mathrm{~d}$ & $12,7 \mathrm{c}$ \\
Baixa & $246,2 \mathrm{~b}$ & $399,0 \mathrm{a}$ & 2256,2 & $1094,4 \mathrm{a}$ & $1,6 \mathrm{~b}$ & $9,2 \mathrm{c}$ & $4,5 \mathrm{ab}$ & $10,8 \mathrm{c}$ & $15,3 \mathrm{~b}$ \\
Moderada & $188,8 \mathrm{c}$ & $353,5 \mathrm{~b}$ & 2099,9 & $854,0 \mathrm{~b}$ & $1,9 \mathrm{a}$ & $11,4 \mathrm{~b}$ & $4,6 \mathrm{a}$ & $13,3 \mathrm{~b}$ & $17,9 \mathrm{a}$ \\
Alta & $181,8 \mathrm{c}$ & $342,0 \mathrm{~b}$ & 2326,5 & $708,2 \mathrm{c}$ & $1,9 \mathrm{a}$ & $13,0 \mathrm{a}$ & $4,0 \mathrm{~b}$ & $15,0 \mathrm{a}$ & $18,9 \mathrm{a}$ \\
\hline CV (\%) & 24,7 & 14,2 & 14,8 & 10,6 & 24,3 & 28,2 & 18,7 & 26,9 & 22,0 \\
\hline
\end{tabular}

${ }^{1}$ Severidade: nula: 0 lesão/fruto; baixa: 1-2 lesões/fruto; moderada: 3-4 lesões/fruto; e alta: 5-16 lesões/fruto.

${ }^{2}$ Médias seguidas de letras distintas diferem entre si, pelo teste de Tukey $(P \leq 0,05)$.

ns: diferença não significativa $(P \geq 0,05)$.

TABELA 2 - Coeficiente da taxa de discriminação paralela (TDP) para a função canônica discriminante $1\left(\mathrm{FCD}_{1}\right)$, referente às análises dos elementos minerais, e suas relações, na casca e na polpa em maçãs 'Fuji'.

\section{Atributos avaliados}

TDP

\begin{tabular}{lcc}
\cline { 2 - 3 } Atributos avaliados & Casca & Polpa \\
\hline $\mathrm{Ca}$ & $\mathbf{0 , 6 4 7 5}$ & $-0,0577$ \\
$\mathrm{~K} / \mathrm{Ca}$ & 0,0873 & 0,1069 \\
$\mathrm{Mg} / \mathrm{Ca}$ & 0,2288 & 0,0183 \\
$\mathrm{~N} / \mathrm{Ca}$ & $-0,0162$ & $-0,0149$ \\
$(\mathrm{~K}+\mathrm{Mg}) / \mathrm{Ca}$ & 0,0000 & 0,0000 \\
$(\mathrm{~K}+\mathrm{Mg}+\mathrm{N}) / \mathrm{Ca}$ & 0,0000 & 0,0000 \\
\hline
\end{tabular}

TABELA 3 - Valores médios dos coeficientes canônicos padronizados (CCP) para a função canônica discriminante $1\left(\mathrm{FCD}_{1}\right)$, referente às análises minerais de maçãs 'Fuji' com diferentes níveis de severidade de "bitter pit".

\begin{tabular}{lc}
\hline Severidade $^{1}$ & CCP \\
\hline Nula & $3,2848 \mathrm{a}^{2}$ \\
Baixa & $1,0374 \mathrm{~b}$ \\
Moderada & $-1,2847 \mathrm{c}$ \\
Alta & $-3,0375 \mathrm{~d}$ \\
\hline
\end{tabular}

${ }^{1}$ Severidade nula: nenhuma lesão/fruto; baixa: 1-2 lesões/fruto; moderada: 3-4 lesões/fruto; e alta: 5-16 lesões/fruto.

${ }^{2}$ Médias seguidas de letras distintas diferem entre si, pelo teste de Tukey $(P \leq 0,05)$. 


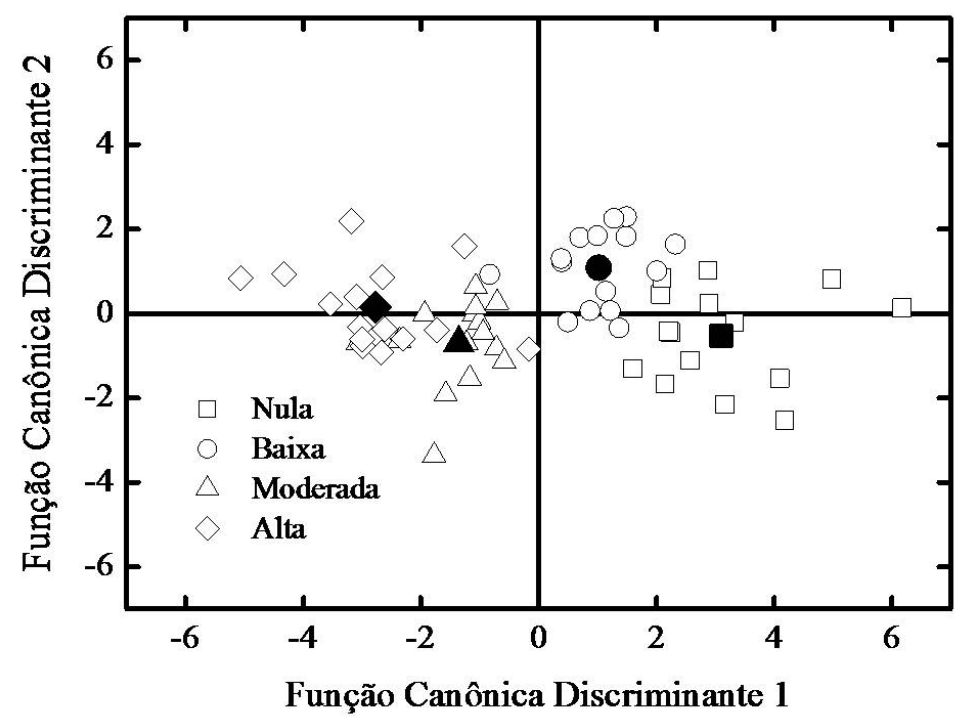

FIGURA 1- Coeficientes canônicos padronizados (CCP) das funções canônicas discriminantes 1 e 2, em maçãs 'Fuji' com diferentes níveis de severidade de "bitter pit", considerando os atributos minerais avaliados na polpa e na casca. Símbolo cheio representa o valor médio de CCP para cada nível de severidade de "bitter pit".

\section{Considerações finais}

A casca apresentou, em relação à polpa, teores de $\mathrm{Ca}, \mathrm{Mg}, \mathrm{K}$ e $\mathrm{N}$ em torno de oito, cinco, duas e quatro vezes maiores, respectivamente (Tabela 1). Como o aumento nos teores minerais na casca em relação à polpa foi maior para o $\mathrm{Ca}$, comparativamente aos demais elementos $(\mathrm{K}, \mathrm{Mg}$ e $\mathrm{N})$, os valores das relações $\mathrm{Mg} / \mathrm{Ca}, \mathrm{K} / \mathrm{Ca},(\mathrm{K}+\mathrm{Mg}) /$ $\mathrm{Ca}, \mathrm{N} / \mathrm{Ca}$ e $(\mathrm{K}+\mathrm{Mg}+\mathrm{N}) / \mathrm{Ca}$ foram menores na casca do que na polpa. A casca apresentou também menores coeficientes de variação $(\mathrm{CV})$ para todos os elementos $(\mathrm{Ca}, \mathrm{Mg}, \mathrm{K}$ e $\mathrm{N})$, e para as relações minerais quantificadas, em relação à polpa. $\mathrm{O}$ maior CV observado na polpa parece ser o resultado da grande variabilidade existente entre frutos, bem como reflexo da amostragem de tecidos na polpa com grandes diferenças nos teores de todos os elementos (LEWIS; MARTIN, 1973; TERBLANCHE et al., 1979; FERGUSON; WATKINS, 1983). Por outro lado, a amostragem da casca reduziu a variabilidade entre frutos e aumentou as diferenças no teor de minerais entre as classes de severidade ao "bitter pit”. Em razão disso, o teor de Ca na casca deve ser utilizado para predição da severidade de "bitter pit" em maçãs 'Fuji'.

\section{CONCLUSÕES}

1- O teor de cálcio na casca é o melhor indicativo da suscetibilidade ao "bitter pit" em maçãs 'Fuji'.

2- Maçãs 'Fuji' com teores de Ca na casca $\leq 240 \mathrm{mg} \mathrm{kg}^{-1}$ de matéria fresca são suscetíveis ao "bitter pit".

\section{REFERÊNCIAS}

AMARANTE, C.V.T. do; CHAVES, D.V.; ERNANI, P.R. Análise multivariada de atributos nutricionais associados ao "bitter pit" em maçãs 'Gala'. Pesquisa Agropecuária Brasileira, Brasília, v.41, n.5, p.841846, 2006a.

AMARANTE, C.V.T. do; CHAVES, D.V.; ERNANI, P.R. Composição mineral e severidade de "bitter pit” em maçãs 'Catarina'. Revista Brasileira de Fruticultura, Jaboticabal, v.28, n.1, p.51-54, 2006 b.

AMARANTE, C.V.T. do; ERNANI, P.R.; CHAVES, D.V. Fruit infiltration with Magnesium is a feasible way to predict bitter pit susceptibility in 'Gala' apples grown in Southern Brazil. Acta Horticulturae, Leuven, n.682, p.1271-1274, 2005. 
ARGENTA, L.C.; SUZUKI, A. Relação entre teores minerais e frequência de bitter pit em maçã cv. Gala no Brasil. Revista Brasileira de Fruticultura, Jaboticabal, v.16, n.1, p.267-277, 1994.

FAUST, M.; SHEAR, C.B. Corking disorders of apple: a physiological and biochemical review. Botanical Review, New York, v.34, p.441-469, 1968.

FERGUSON, I.B.; WATKINS, C.B. Cation distribution and balance in apple fruit in relation to calcium treatments for bitter pit. Scientia Horticulturae, Amsterdam, v.19, n.3/4, p.301-310, 1983.

FERGUSON, I.B; WATKINS, C.B. Bitter-pit in apple fruit. Horticultural Reviews, New York, v.11, p.289-355, 1989.

LEWIS, T.L.; MARTIN, D. Longitudinal distribution of applied calcium, and of naturally occurring calcium, magnesium, and potassium in Merton apple fruits. Australian Journal of Agricultural Research, Collingwood, v.24, n.3, p.363-371, 1973.

NACHTIGALL, G.R.; FREIRE, C.J.S. Previsão da incidência de "bitter pit" em maçãs através dos teores de cálcio em folhas e frutos. Revista Brasileira de Fruticultura, Jaboticabal, v.20, n.2, p.158-166, 1998.

POOVAIAH, B.W. Role of calcium in prolonging storage life of fruits and vegetables. Food Technology, Chicago, v.40, n.1, p.86-89, 1986.
POOVAIAH, B.W.; GLENN, G.M.; REDDY, A.S.N. Calcium and fruit softening: physiology and biochemistry. Horticultural Reviews, New York, v.10, p.107-152, 1988.

SAS INSTITUTE. Getting started with the SAS learning edition. Cary: SAS, 2002. 200p.

SAURE, M.C. Calcium translocation to fleshy fruit: its mechanism and endogenous control. Scientia Horticulturae, Amsterdam, v.105, n.1, p.65-89, 2005.

TEDESCO, M.J.; GIANELLO, C.; BISSANI, C.A.; BOHNEN, H.; VOLKWEISS, S.J. Análise do solo, planta e outros materiais. 2.ed, Porto Alegre: Departamento de Solos, UFRGS, 1995. 174 p. (Boletim Técnico de Solos, 5).

TERBLANCHE, J.H. An integrated approach to orchard nutrition and bitter pit control. Deciduous Fruit Grower, Belville, v.31, n.12, p.501-513, 1981.

TERBLANCHE, J.H.; GÜRGEN, K.H.; PEINAR,W.J. Concentration gradients of $\mathrm{K}, \mathrm{Ca}$ and $\mathrm{Mg}$ in Golden Delicious apples with reference to bitter pit. Deciduous Fruit Grower, Belville, v.29, n.2, p.76-79, 1979. 